\section{Impacto da Estratégia Saúde da Família com equipe de saúde bucal sobre a utilização de serviços odontológicos}

\author{
Inclusion of oral health teams in the Family \\ Health Program and its impact on the use of \\ dental services
}

Carmen Regina dos Santos Pereira 1

Alberto Allan Rodrigues Patrício ${ }^{2}$

Fábio Andrey da Costa Araújo 2

Eudes Euler de Souza Lucena ${ }^{2}$

Kenio Costa de Lima 2

Angelo Giuseppe Roncalli 2

\footnotetext{
1 Programa de Pós-graduação em Ciências da Saúde, Universidade Federal do Rio Grande do Norte, Natal, Brasil.

2 Programa de Pós-graduação em Odontologia, Universidade Federal do Rio Grande do Norte, Natal, Brasil.

Correspondência C. R. S. Pereira Programa de Pós-graduação em Ciências da Saúde, Universidade Federal do Rio Grande do Norte. Rua Romualdo Galvão 3673 apto. 404H, Natal, RN 59056-100, Brasil. carmenpuc@ig.com.br
}

\begin{abstract}
The aim of this study was to determine whether the incorporation of Oral Health Teams (OHT) into the Family Health Program (FHP) led to greater use of dental services in Natal, Rio Grande do Norte State, Brazil. Twenty-two census tracts were studied, 11 located in areas covered by the FHP for at least one year and paired according to socioeconomic criteria with 11 tracts in areas without coverage. The final sample consisted of 4,604 subjects. The areas covered by OHT in the FHP (model 1) were compared to areas covered by the FHP but without OHT (model 2) and to areas covered by traditional primary care services or without any coverage (model 3). In areas covered by model 1,32\% of the population used dental care, whereas in models 2 and 3 the rates were $45.4 \%$ and $17.5 \%$, respectively $(p<0.001)$. With respect to age, model 1 only exceeded model 2 in the age bracket 12 years and under. The study concludes that OHT in the FHP only had a positive impact on the target indicator when compared to model 3.
\end{abstract}

Health Services Evaluation; Family Health Program; Oral Healh

\section{Introdução}

O Programa Saúde da Família (PSF), estratégia adotada em nível nacional desde 1994, tem como principal propósito a organização da prática da atenção à saúde sob novas bases, centrando o foco na família, na tentativa de alcançar uma melhoria na qualidade de vida dos brasileiros.

A implementação dessa estratégia, considerada eixo estruturante da atenção básica, embora sob os preceitos do Sistema Único de Saúde (SUS), vem produzindo experiências qualitativamente diferentes e, em muitos casos, reproduzindo o modelo tradicional em novas embalagens 1,2. Na avaliação de Ronzani \& Stralen ${ }^{3}$ não há uma atuação uniforme das equipes do PSF, de forma que, mesmo com um discurso travestido de novo, o modelo de atenção individual curativa continua se perpetuando de forma hegemônica.

No que se refere à saúde bucal, o Ministério da Saúde publicou a Portaria GM/MS no $n^{\circ} .444$, em 2000 , estabelecendo o incentivo financeiro para a reorganização da atenção à saúde bucal prestada nos municípios por meio do PSF, incluindo desta maneira as ações de saúde bucal no PSF na tentativa de se alcançar este modo integral de atenção, tomando-a como parte integrante da saúde das famílias e comunidades 5,6. Em todo esse tempo, desde que foi estabelecido o incentivo para a implantação das equipes de saúde bucal no PSF, houve um crescimento exponencial do número de equipes em todo o Brasil. De acordo com os 
dados mais recentemente disponibilizados pelo Ministério da Saúde, um total de 16.190 equipes de saúde bucal estavam implantadas em todo o território nacional até janeiro de 2008. Esse número era 4.261 em 2002, indicando um crescimento de $280 \%$ em cinco anos 7 . Apesar disso, pouco tem sido feito no sentido de monitorar tal inclusão e avaliar seus impactos, o que pode ser constatado pela escassa literatura sobre este tema. Chaves \& Vieira-da-Silva 8 em sua revisão ressaltam que há poucos estudos sobre como se desenvolvem as práticas de saúde bucal nos serviços odontológicos públicos no Brasil 9,10,11. Os trabalhos de Serra 12, Roncalli 9 e Peres 10 , desenvolvidos nos anos 1990, investigaram algumas intervenções municipais exitosas de reorganização das ações odontológicas no setor público em Curitiba (Paraná), Campinas (São Paulo), Belo Horizonte e Ipatinga (Minas Gerais). Nesses casos, o principal fator relacionado ao êxito tem sido o apoio do governo local 9,11. Esses estudos apontam para a importância da investigação também dos componentes da gestão municipal mais favoráveis para o desenvolvimento da atenção à saúde bucal pública do país 9,10,12 .

$\mathrm{Na}$ verdade, os processos de avaliação e monitoramento dentro dos serviços de saúde são amiúde raros. Segundo Bodstein et al. 13 a avaliação em saúde ganha relevância a partir de 2000 em torno da metodologia de avaliação de desempenho proposta pela Organização Mundial da Saúde (OMS). No Brasil, os estudos de linha de base iniciados a partir de 2005 aparecem como um componente importante para o processo de institucionalização da avaliação em saúde do país 13 .

No campo da saúde bucal tais estudos ainda são menos freqüentes. Baldani et al. 14 analisaram o perfil de implantação da odontologia no PSF em 136 municípios do Estado do Paraná um ano após a vigência da Portaria GM/MS no. 1.4444 e encontraram pontos positivos no sentido da recepção favorável por parte da população e participação dos cirurgiões-dentistas em cursos de capacitação, e já antecipavam a questão do nó crítico do encaminhamento dos casos complexos e a necessidade de formação de profissionais generalistas com perfil adequado.

Andrade \& Ferreira 15 e Oliveira \& Saliba 16 também identificaram lacunas na implantação da saúde bucal nos municípios avaliados. No entanto, tais estratégias são extremamente importantes para uma adequada condução das ações de saúde.

O processo de monitoramento, segundo Silva Jr. \& Mascarenhas 1, necessita ser complementado com a abordagem da pesquisa avaliativa na busca da percepção dos movimentos de mu- danças na qualidade da atenção no que se refere, principalmente, ao processo de trabalho das equipes, na relação com as populações adscritas e à integralidade da atenção à saúde. Portanto, resultados de ações e programas de saúde podem ser avaliados com relação às mudanças verificadas, tanto no estado de saúde das populações, como em termos de conhecimentos e comportamentos derivados das práticas desenvolvidas 17 .

A pesquisa de avaliação, que tem como objeto uma dada intervenção na realidade social, trata de avaliar a política pública, entendida, segundo Draibe 18 , como a que se desenvolve em esferas públicas da sociedade e não no plano privado e interno das instituições ou organizações da sociedade.

Por meio da avaliação de resultados, consegue-se pesquisar se determinados programas cumpriram ou estão cumprindo seus objetivos. Ainda de acordo com Draibe ${ }^{18}$, esse tipo de avaliação é também denominado de avaliação de impacto. Os impactos referem-se às alterações ou mudanças efetivas na realidade sobre a qual um programa intervém e por ele são provocadas. Os indicadores de impacto devem ser, portanto, capazes de aferir os efeitos líquidos do programa sobre a população alvo.

Desde a publicação da Pesquisa Mundial de Saúde 19, que colocou em evidência as desigualdades em saúde na população brasileira, estudos locais vêm comprovando a associação entre as condições de vida e o acesso a serviços públicos de saúde. No tocante à saúde bucal, alguns estudos já realizados claramente demonstram um aumento da oferta de serviços odontológicos em áreas cobertas pelo PSF, sem, no entanto, se traduzir em reversão do modelo de atenção à saúde bucal, caracterizado por ser pouco resolutivo, mutilador e, portanto, centrado na doença 20,21,22.

Do ponto de vista conceitual, percebe-se que, apesar das discordâncias na terminologia e na abrangência dos conceitos de utilização e de acesso, podem ser traçadas algumas linhas de concordância entre os autores. Quanto à terminologia, a acessibilidade é preferida pelos autores que a descrevem como uma característica da oferta de serviços de saúde ou do ajuste entre a oferta e a população, seja esta uma característica geral, seja restrita à acessibilidade geográfica. Já os autores que optam pelo termo acesso, em geral, centram-no na entrada inicial dos serviços de saúde, podendo-se citar, como exemplo, o modelo de Andersen 23. De qualquer forma, prevalece a idéia de que acesso é uma dimensão do desempenho dos sistemas de saúde associada à oferta. Quanto à abrangência do conceito, observa-se uma tendência de ampliação do escopo, 
com deslocamento do seu eixo da entrada nos serviços para os resultados dos cuidados recebidos ${ }^{23}$. No presente estudo, será tomado como referência o termo "utilização" ou "uso" de serviços de saúde, considerando que foi avaliada a freqüência de ida ao dentista no setor público em relação à morbidade dentária referida.

Desse modo, tendo em vista a necessidade de se avaliar os programas implementados pela atual política vigente e a complexidade de implantá-los em grandes centros urbanos que abrigam populações com diferentes níveis sócio-econômicos, o objetivo do presente estudo foi verificar se a incorporação da equipe de saúde bucal no PSF gerou uma maior utilização dos serviços odontológicos no Município de Natal, Rio Grande do Norte, Brasil.

\section{Metodologia}

\section{Tipo de estudo}

Trata-se de um ensaio comunitário em paralelo, quase-randomizado. Na tipologia dos estudos epidemiológicos, constitui-se num estudo individuado do ponto de vista do seu tipo operativo, e cuja intervenção, implantação da equipe de saúde bucal já foi realizada. Seus efeitos, portanto, foram avaliados no tempo atual, o que caracteriza um estudo longitudinal. É um estudo em paralelo, no qual um grupo é classificado como sendo alvo da intervenção (equipe de saúde bucal - PSF) e outro não 24 .

\section{Plano amostral}

\section{- Definição das unidades amostrais}

Por se tratar de um conjunto de dados obtidos por meio de uma amostra complexa, que combina setores censitários (primeiro estágio) e, dentro dos setores, os domicílios (segundo estágio) é fundamental buscar a homogeneidade entre as áreas nas quais temos a intervenção (áreas cobertas) e as áreas onde ela não se faz presente (não-cobertas). Assim, de algum modo, foram necessários dados relativos às áreas cobertas $\mathrm{e}$ não-cobertas no sentido de agrupá-los e gerar critérios de emparelhamento das mesmas.

Uma unidade de análise que contém informações relevantes para esse fim em todas as áreas, independente de cobertura, é o setor censitário, o qual se constitui na menor unidade de análise utilizada pelo Instituto Brasileiro de Geografia e Estatística (IBGE) para as quais se pode dispor de dados sócio-econômicos, constando de aglomerados de, em média, 300 domicílios.
Pelo fato do PSF trabalhar com a idéia de microáreas e não de setor censitário, em sua territorialização, foi necessário transferir as informações sobre as microáreas de atuação das equipes coletadas em cada unidade de saúde para o mapa da cidade de Natal, construído com base nos cartogramas do IBGE, determinando quais setores censitários faziam parte das áreas cobertas e não-cobertas pelas equipes de saúde bucal.

Da totalidade de setores, foram excluídos aqueles que estavam parcialmente inseridos em áreas cobertas pelas equipes de saúde bucal. Portanto, somente aqueles totalmente inseridos em áreas cobertas e não-cobertas compuseram o universo a partir do qual foram estabelecidos os grupos.

Uma vez estabelecido o universo a ser incluído, foram sorteados 15 setores das áreas cobertas pelas equipes de saúde bucal, os quais compuseram as áreas sob intervenção. Para compor as áreas de não intervenção (controles), foi realizado um emparelhamento intencional valendose dos dados constantes nas bases de dados dos setores censitários.

Esses dados são relativos às principais variáveis as quais, sabidamente, exercem influência sobre as variáveis de estudo, entre elas as de natureza sócio-econômica e sanitária. A partir da Base de Dados por Setor Censitário 25, disponibilizada pelo IBGE, foram selecionadas algumas variáveis sócio-econômicas, as quais compuseram um índice classificatório final 26 , para que o emparelhamento pudesse ser realizado.

Desse modo, ficou garantido o controle dos vieses relativos às variáveis citadas. Além disso, a randomização realizada para a alocação dos setores do grupo experimental (cobertos) possibilitou que diferentes pontos em toda a área do município fossem contemplados. O emparelhamento intencional também buscou manter essa distribuição geográfica, de modo que, para cada par "experimental/controle" tivesse não só a mesma classificação, mas uma semelhança geográfica, como se observa na Figura 1.

\section{- Variáveis}

A variável de interesse explanatória foi a presença da equipe de saúde bucal no PSF. A variável resposta (dependente) foi o acesso à assistência odontológica pública, entendida como a proporção de indivíduos que foram consultados por cirurgião-dentista da rede pública nos 12 meses anteriores à pesquisa, associada à necessidade referida de tratamento dentário.

As variáveis independentes de confusão foram obtidas em nível da família (domicílio). A Ta- 
Figura 1

Distribuição dos setores experimentais e controles do Município de Natal, Rio Grande do Norte, Brasil, 2006

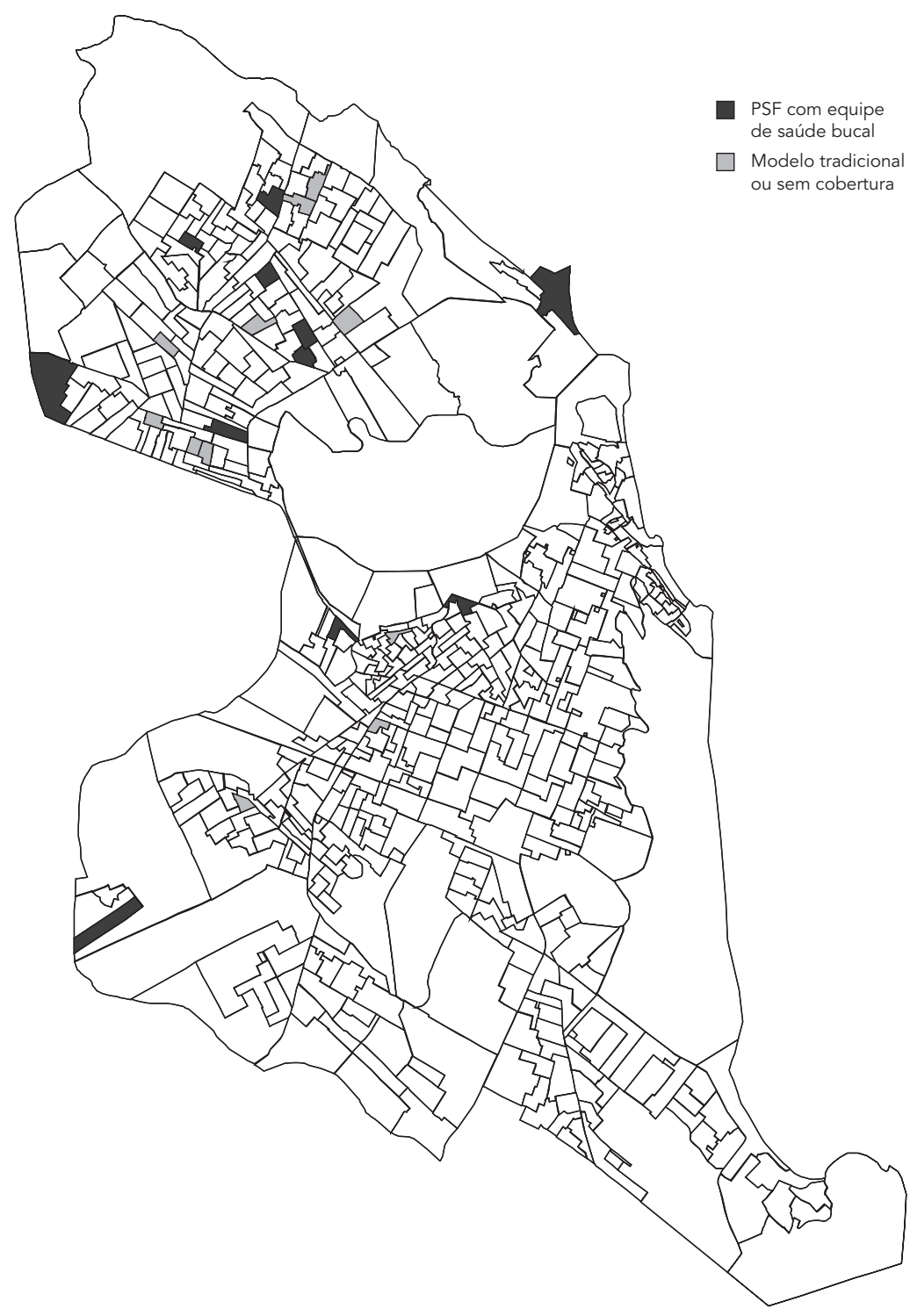

PSF: Programa Saúde da Família.
Pesquisa Nacional por Amostra de Domicílios 2003 (PNAD 2003) 27, realizada pelo IBGE e o Ministério da Saúde. A PNAD 2003 apresenta, entre outros dados, o perfil das necessidades em saúde no país, com base em declarações sobre a auto-avaliação do estado de saúde das pessoas, bem como sobre o acesso e utilização dos serviços e atendimento no SUS, parâmetros de interesse do presente trabalho.

De acordo com este estudo, cerca de $38 \%$ da população avaliada teve acesso à assistência odontológica nos 12 meses anteriores à pesquisa. Supondo um efeito presumido da intervenção (neste caso, a implantação das equipes de saúde bucal) de $20 \%$, associado a um nível de significância de 0,05 e um poder de 95\%, e de acordo com a técnica preconizada por Altman 28 e também utilizada por Roncalli \& Lima 26 , chegou-se a um número de 2.300 indivíduos em cada grupo.

Considerando uma taxa de não-resposta de $20 \%$ e um efeito de desenho de 1,5, a amostra requerida passou a ser de 4.140 indivíduos em cada grupo, totalizando 8.280 pessoas.

\section{- Coleta dos dados}

Foi desenvolvida uma ficha a ser aplicada pelos auxiliares de consultório dentário nas residências. Esse questionário-entrevista foi aplicado junto ao informante mais qualificado, sendo, portanto, um instrumento que agrega informações relativas a todos os residentes no domicílio.

Os auxiliares de consultório dentário do próprio serviço foram treinados em oficinas de trabalho de 12 horas de duração (três turnos), associando a familiarização com os instrumentos a um treinamento em campo. No primeiro turno, foi feita a discussão dos objetivos do estudo e suas principais características. Em seguida, o treinamento propriamente dito foi realizado a partir da discussão do manual de instruções e da ficha de coleta, previamente validada, além do reconhecimento dos mapas dos setores e identificação das áreas de trabalho e dos percursos. No segundo turno (tarde), os auxiliares de consultório dentário fizeram um treinamento em suas áreas de atuação e, no terceiro turno, foram esclarecidas todas as dúvidas a respeito do exercício e estabelecidos os encaminhamentos e prazos da pesquisa.

\section{- Análise dos dados}

Para o cálculo do tamanho da amostra deste estudo, tomou-se como base a proporção de indivíduos que tiveram acesso à assistência odontológica no ano anterior, segundo os dados da

bela 1 traz a descrição dessas variáveis que foram utilizadas na análise.

\section{- Tamanho da amostra}

O banco de dados da pesquisa foi construído em programa especificamente desenvolvido para a entrada dos dados, com posterior verificação de 
Elenco de variáveis independentes de confusão para o estudo.

\begin{tabular}{|c|c|}
\hline Variável & Descrição \\
\hline Aglomeração domiciliar & $\begin{array}{l}\text { Número de indivíduos por cômodo no domicílio dicotomizados a partir da mediana, } \\
\text { formando as categorias: "desfavorável" }(<0,80) \text { e "favorável" }(\geq 0,80)\end{array}$ \\
\hline Renda familiar & $\begin{array}{l}\text { Renda em reais do chefe da família no último mês dicotomizados a partir da mediana, } \\
\text { formando as categorias: "desfavorável" }(<\mathrm{R} \$ 500,00) \text { e "favorável" }(\geq \mathrm{R} \$ 500,00)\end{array}$ \\
\hline Escolaridade da mãe & $\begin{array}{l}\text { Anos de estudo da mãe dicotomizados a partir da mediana, } \\
\text { formando as categorias: "desfavorável" (< } 4 \text { anos) e "favorável" ( } \geq 4 \text { anos) }\end{array}$ \\
\hline
\end{tabular}

consistência da digitação. Considerando o modelo teórico do estudo, as variáveis independentes e o desfecho foram devidamente trabalhados no sentido de comporem um modelo de análise estratificada. Nesse sentido, os efeitos da intervenção, ajustados para todas as variáveis de confusão, foram observados no principal desfecho com base no teste do qui-quadrado e sua magnitude verificada pelo cálculo da razão de prevalência (RP), ajustada para cada estrato e seus respectivos intervalos de confiança. Considerouse como nível de significância para este estudo o limite de $\alpha=0,05$.

\section{a) Descrição da estratégia de análise}

A análise da assistência odontológica, considerada como variável dependente principal (desfecho), foi baseada nas questões: "Acha que necessita de tratamento dentário atualmente?" e "Foi ao cirurgião-dentista da sua unidade de saúde?”. A nova variável, criada baseando-se nessas informações, se refere à utilização do programa de saúde bucal para os indivíduos que responderam positivamente a essas questões (achavam que tinham necessidade de tratamento dentário e foram ao cirurgião-dentista da rede pública) ou que responderam sim à primeira questão e não à segunda (achavam que tinham necessidade, mas não foram ao cirurgião-dentista da rede pública), formando as categorias "assistido" e "não-assistido" da amostra estudada.

É importante ressaltar que, no Município de Natal, à época da coleta dos dados, todas as equipes de saúde bucal eram constituídas de cirurgiões-dentistas e auxiliares de consultório dentário (modalidade 1). A inserção dessas equipe de saúde bucal ocorreu, como em outros municípios, de forma gradual, gerando situações do tipo: equipes de saúde bucal lotadas e trabalhando integradas ao PSF, cirurgiões-dentistas em unidades com Programa de Agentes
Comunitários de Saúde (PACS) ou PSF mas não integrados a esse novo processo de trabalho e, por fim, consultórios dentários nas unidades básicas de saúde (UBS) sem a presença do PACS ou PSF. Portanto, por essa particularidade, na análise dos dados com relação à variável independente principal, o tipo de cobertura foi dividido em três modelos:

i) Modelo 1: se caracteriza pela efetiva implantação do Programa de Saúde Bucal no PSF (SB-PSF) com tempo mínimo de um ano (o tempo médio de cobertura encontrado para as áreas cobertas pela equipe de saúde bucal foi de 3,22 anos). Neste estudo foi considerado como grupo experimental;

ii) Modelo 2: se caracteriza pela existência de cirurgiões-dentistas, lotados em unidades com PSF ou PACS, mas sem vinculação a um ou outro modelo assistencial, trabalhando numa organização de trabalho "não-PSF". Neste estudo, será considerado um modelo de transição e comporá um dos grupos controle;

iii) Modelo 3: se caracteriza pela presença de UBS tradicional (sem PACS nem PSF), que mantém um cirurgião-dentista trabalhando também numa organização de trabalho "não-PSF". Esse modelo será considerado no contexto do estudo, nível mínimo de organização de serviço. Também fazem parte desse modelo as demais áreas sem cobertura. Para efeito de análise, será considerado como segundo grupo controle.

Para verificação do possível efeito de variáveis de confusão relacionadas às condições sócio-econômicas do domicílio, optou-se pela estratégia de análise estratificada. As variáveis "aglomeração domiciliar", "renda familiar" e "escolaridade da mãe", devidamente dicotomizadas, foram incluídas no modelo com o intuito de verificar possíveis efeitos de confusão e interação de acordo com a técnica descrita por Luiz 29. 


\section{- Aspectos éticos}

O projeto de pesquisa foi submetido ao Comitê de Ética em Pesquisa da Universidade Federal do Rio Grande do Norte para a devida análise, recebendo parecer favorável à sua execução, estando registrado no Sistema Nacional de Ética em Pesquisa (SISNEP) sob o número 0028.1.051.00006.033/2006, apresentando-se de acordo com a Resolução $n^{o}$. 196/96 do Conselho Nacional de Saúde.

Figura 2

Descrição da amostra do estudo. Natal, Rio Grande do Norte, Brasil, 2006.

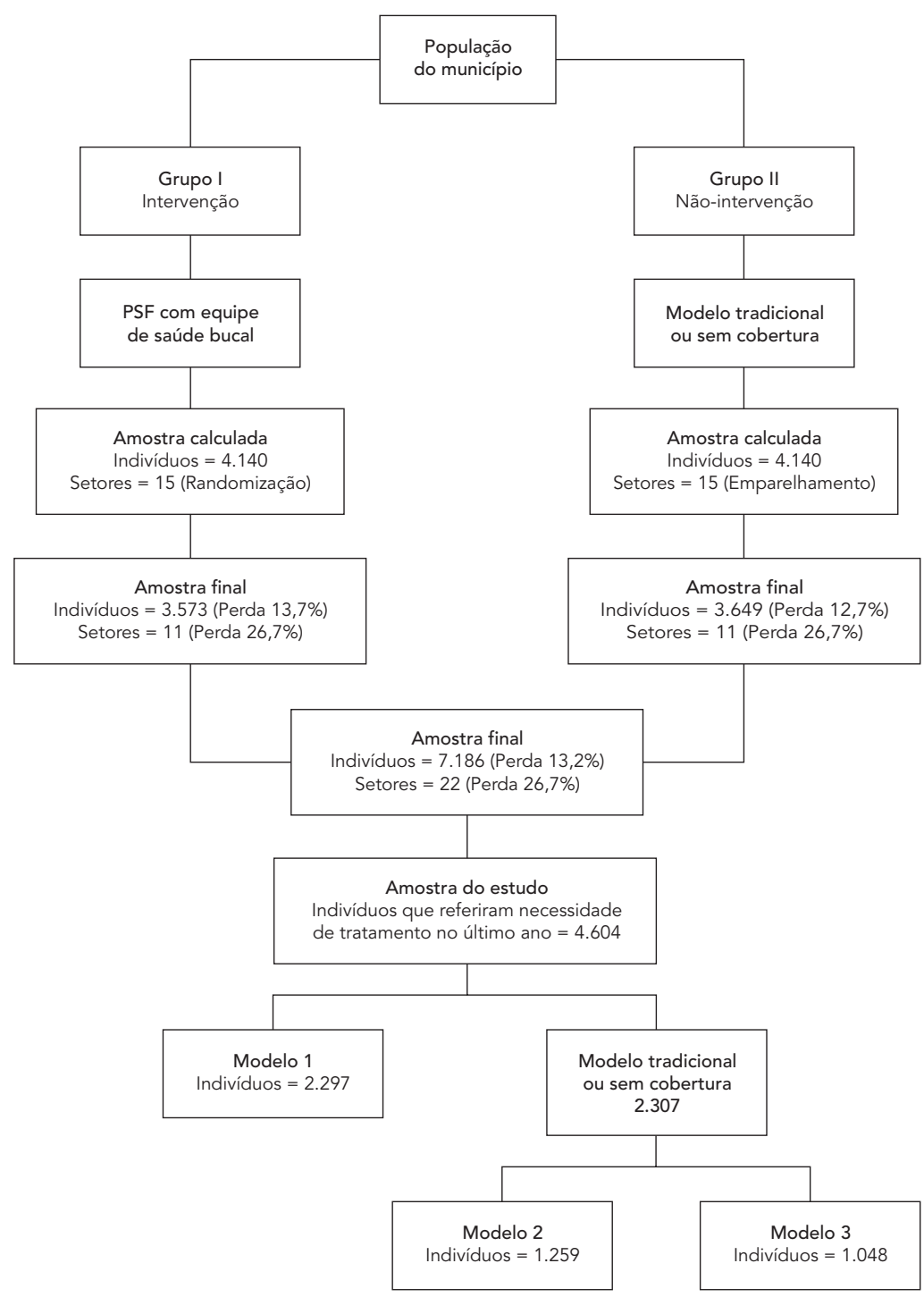

PSF: Programa Saúde da Família

\section{Resultados}

\section{Descrição da amostra}

Dos 15 setores previstos para compor cada grupo, de acordo com a descrição da amostra na Figura 2, alguns setores, quatro em áreas cobertas pelo modelo SB-PSF, e conseqüentemente, os quatro setores controle, deixaram de ser investigados devido a problemas operacionais. A análise, portanto, se refere aos dados coletados em 22 setores, sendo 11 em cada grupo, perfazendo um total de 7.186 indivíduos. A perda final $(13,2 \%)$ está dentro dos limites esperados para o desenho do estudo proposto, o qual previu uma taxa de não-resposta de $20 \%$.

Do total de indivíduos entrevistados, 4.604 referiram necessidade de tratamento no último ano. Desse universo, 2.297 entrevistados pertenciam às áreas com cobertura do SB-PSF e o restante às áreas com modelo tradicional ou sem cobertura.

\section{Avaliação do impacto da SB-PSF sobre a assistência odontológica}

\section{- Análise do impacto em função da idade}

A Tabela 1 apresenta as freqüências dos indivíduos assistidos segundo a modalidade de cobertura e de acordo com as faixas etárias. Verifica-se, na análise entre as categorias de assistência odontológica, que as diferenças entre os modelos foram significativas em todas as faixas etárias, tendo como referência o modelo 1 .

Os grupos de adolescentes, adultos e idosos apresentaram um percentual maior de indivíduos atendidos no modelo 2, considerado, no estudo, um modelo tradicional. Somente a faixa etária menor de 12 anos apresentou uma inversão, em que o modelo 1 (SB-PSF) ultrapassa o modelo 2 .

O percentual de entrevistados que compõem o grupo "não-assistido", independentemente do modelo de assistência odontológica, é superior a $63 \%$, conforme mostrado na Tabela 2.

\section{- Análise considerando potenciais fatores de confusão}

As variáveis "aglomeração domiciliar", "escolaridade da mãe" e "renda familiar" foram avaliadas em relação à variável desfecho e somente a primeira apresentou associação significativa $(\mathrm{p}<$ $0,001)$. Nesse sentido, essa foi a única variável incluída na análise estratificada. Foram calculadas as RP bruta e ajustada para assistência odontológica, segundo o tipo de cobertura, considerando 
Distribuição, em números absolutos e percentuais, da assistência odontológica segundo modalidade de cobertura e faixa etária. Natal, Rio Grande do Norte, Brasil, 2006

\begin{tabular}{|c|c|c|c|c|c|c|c|c|c|c|}
\hline \multirow[t]{3}{*}{ Grupo etário } & \multicolumn{8}{|c|}{ Cobertura de saúde bucal } & \multicolumn{2}{|c|}{ Total } \\
\hline & \multicolumn{2}{|c|}{ Modelo 1} & \multicolumn{2}{|c|}{ Modelo 2} & \multirow[t]{2}{*}{ Valor de p * } & \multicolumn{2}{|c|}{ Modelo 3} & \multirow[t]{2}{*}{ Valor de $p$ ** } & \multirow[b]{2}{*}{$\mathbf{n}$} & \multirow[b]{2}{*}{$\%$} \\
\hline & $\mathrm{n}$ & $\%$ & $\mathrm{n}$ & $\%$ & & $\mathrm{n}$ & $\%$ & & & \\
\hline \multicolumn{11}{|c|}{ Crianças (< 12 anos) } \\
\hline Assistido & 212 & 42,2 & 77 & 33,6 & 0,035 & 38 & 18,7 & $<0,001$ & 327 & 35,0 \\
\hline Não-assistido & 291 & 57,8 & 152 & 66,4 & & 163 & 81,3 & & 606 & 65,0 \\
\hline Total & 503 & 100,0 & 229 & 100,0 & & 201 & 100,0 & & 933 & 100,0 \\
\hline \multicolumn{11}{|c|}{ Adolescentes (13 a 19 anos) } \\
\hline Assistido & 139 & 36,7 & 94 & 45,6 & 0,043 & 48 & 26,4 & 0,018 & 285 & 37,1 \\
\hline Não-assistido & 240 & 63,3 & 112 & 54,4 & & 135 & 73,6 & & 483 & 62,9 \\
\hline Total & 379 & 100,0 & 206 & 100,0 & & 183 & 100,0 & & 768 & 100,0 \\
\hline \multicolumn{11}{|c|}{ Adultos (20 a 59 anos) } \\
\hline Assistido & 342 & 26,6 & 362 & 48,3 & $<0,001$ & 87 & 14,5 & $<0,001$ & 805 & 30,5 \\
\hline Não-assistido & 945 & 73,4 & 387 & 51,7 & & 516 & 85,5 & & 1.834 & 69,5 \\
\hline Total & 1.287 & 100,0 & 749 & 100,0 & & 603 & 100,0 & & 2.639 & 100,0 \\
\hline \multicolumn{11}{|c|}{ Idosos (60 anos e mais) } \\
\hline Assistido & 42 & 32,6 & 41 & 54,5 & 0,004 & 11 & 17,5 & 0,048 & 92 & 35,0 \\
\hline Não-assistido & 85 & 66,4 & 34 & 45,5 & & 50 & 82,5 & & 172 & 65,0 \\
\hline Total & 128 & 100,0 & 75 & 100,0 & & 61 & 100,0 & & 264 & 100,0 \\
\hline \multicolumn{11}{|l|}{ Amostra total } \\
\hline Assistido & 733 & 31,9 & 572 & 45,4 & $<0,001$ & 183 & 17,5 & $<0,001$ & 1.506 & 32,7 \\
\hline Não-assistido & 1.564 & 68,1 & 687 & 54,6 & & 865 & 82,5 & & 2.914 & 63,3 \\
\hline Total & 2.297 & 100,0 & 1.259 & 100,0 & & 1.048 & 100,0 & & 4.604 & 100,0 \\
\hline
\end{tabular}

* Valores de p relativos à comparação entre o modelo 1 e modelo 2 (teste de $\chi^{2}$ com correção de Yates);

** Valores de p relativos à comparação entre o modelo 1 e modelo 3 (teste de $\chi^{2}$ com correção de Yates).

esta variável como potencial fator de confusão e interação. O objetivo dessa estratégia foi verificar uma possível associação entre as variáveis, independentemente de condições sócio-econômicas.

De acordo com a Tabela 3, nas áreas com SBPSF (modelo 1) a "desassistência" esteve presente em $68 \%$ dos indivíduos; este percentual foi de $54,6 \%$ nas áreas cobertas pelo modelo 2 . Isso se traduziu em uma razão de prevalência ajustada, quando comparados os dois modelos, de 0,71 (intervalo de 95\% de confiança - IC95\%: 0,650,$77 ; \mathrm{p}<0,001$ ), indicando um "efeito protetor" nas áreas cobertas pelo modelo 2. No entanto, quando se compara o modelo 1 com o modelo 3, a RP para a desassistência foi de 1,84 (IC95\%: $1,60-2,13 ; \mathrm{p}<0,001$ ), indicando maior probabilidade de não obter assistência odontológica para os indivíduos residentes nas áreas relacionadas ao modelo 3, considerado, neste estudo, como modelo tradicional ou sem cobertura.

Ainda de acordo com a Tabela 3, o efeito da variável “aglomeração domiciliar" enquanto pro- vável fator de confusão foi nulo, uma vez que os valores de RP bruta e ajustada são iguais nas duas comparações. Observando-se os valores das RP nos dois estratos de aglomeração domiciliar (favorável e desfavorável), percebe-se que as mesmas não diferem da RP total, indicando ausência de possível efeito de interação. Em outras palavras, o efeito dos distintos modelos de cobertura sobre a variável desfecho (assistência odontológica) pode ser analisado, desconsiderando as interferências das condições sócio-econômicas. Isso, em certa medida, já era esperado, considerando o cuidado em evitar esse tipo de viés pela estratégia descrita anteriormente de emparelhamento dos setores pesquisados.

\section{Discussão}

Medidas de avaliação do acesso e utilização de serviços são importantes indicadores das condições de saúde da população. Em grandes centros, sabidamente, é possível confrontar-se 
Razão de prevalência (RP) para assistência odontológica segundo o tipo de cobertura estudada e aglomeração domiciliar. Natal, Rio Grande do Norte, Brasil, 2007

\begin{tabular}{|c|c|c|c|c|c|c|c|c|c|c|c|}
\hline \multirow{3}{*}{$\begin{array}{l}\text { Aglomeração } \\
\text { domiciliar }\end{array}$} & \multirow[t]{3}{*}{ Cobertura } & \multicolumn{6}{|c|}{ Assistência odontológica pública } & \multirow{3}{*}{$\mathrm{RP}_{\text {bruta }}$} & \multirow[t]{3}{*}{$\mathrm{RP}_{\text {ajustada }}$} & \multirow[t]{3}{*}{ IC95\% } & \multirow{3}{*}{$\begin{array}{l}\text { Valor } \\
\text { de } p\end{array}$} \\
\hline & & \multicolumn{2}{|c|}{ Assistido } & \multicolumn{2}{|c|}{ Não-assistido } & \multicolumn{2}{|c|}{ Total } & & & & \\
\hline & & n & $\%$ & n & $\%$ & n & $\%$ & & & & \\
\hline \multirow[t]{3}{*}{ Favorável $(<0,80)$} & Modelo 1 & 297 & 28,4 & 749 & 71,6 & 1.046 & 100,0 & & 1,00 & & \\
\hline & Modelo 2 & 211 & 40,7 & 307 & 59,3 & 518 & 100,0 & & 0,70 & $0,61-0,88$ & $<0,001$ \\
\hline & Modelo 3 & 71 & 15,7 & 383 & 84,3 & 454 & 100,0 & & 1,82 & $1,44-2,30$ & $<0,001$ \\
\hline \multirow[t]{3}{*}{ Desfavorável $(\geq 0,80)$} & Modelo 1 & 438 & 35,1 & 809 & 64,9 & 1.247 & 100,0 & & 1,00 & & $<0,001$ \\
\hline & Modelo 2 & 361 & 48,9 & 378 & 51,1 & 739 & 100,0 & & 0,72 & $0,65-0,89$ & $<0,001$ \\
\hline & Modelo 3 & 112 & 18,8 & 482 & 81,2 & 594 & 100,0 & & 1,86 & $1,55-2,24$ & $<0,001$ \\
\hline \multirow[t]{3}{*}{ Total } & Modelo 1 & 734 & 32,0 & 1.559 & 68,0 & 2.293 & 100,0 & 1,00 & 1,00 & & $<0,001$ \\
\hline & Modelo 2 & 571 & 45,4 & 686 & 54,6 & 1.257 & 100,0 & 0,70 & 0,71 & $0,65-0,77$ & $<0,001$ \\
\hline & Modelo 3 & 183 & 17,5 & 865 & 82,5 & 1.048 & 100,0 & 1,84 & 1,84 & $1,60-2,13$ & $<0,001$ \\
\hline
\end{tabular}

Modelo 1: unidades com Programa Saúde da Família com equipes de saúde bucal; Modelo 2: unidades com Programa Saúde da Família sem equipes de saúde bucal e unidades básicas de saúde com Programa de Agentes Comunitários de Saúde; Modelo 3: unidade básica de saúde tradicional e áreas descobertas.

com diferentes estratos sócio-econômicos. A estratégia de definição do setor censitário como unidade amostral foi fundamental para que fossem identificados esses diferentes grupos populacionais existentes no município. O uso de indicador classificatório permitiu, por sua vez, que o emparelhamento das áreas cobertas e não-cobertas fosse realizado controlando-se a existência de vieses.

O percentual relativamente pequeno de perdas e recusas, considerando-se as dificuldades operacionais de um estudo desta natureza, deveu-se, em parte, ao investimento no controle do trabalho de campo e no apoio institucional.

No intuito de evitar problemas na análise comparativa, o critério para inclusão de áreas cobertas considerou apenas as áreas onde efetivamente se deu a implantação das equipes de saúde bucal pelo período mínimo de um ano. Trata-se de um corte arbitrário e pode ser considerado pouco tempo para se realizar uma avaliação de impacto. No entanto, os resultados aqui apresentados representam um primeiro esforço de análise e preenchem uma lacuna de conhecimento, uma vez que não havia dados recentes disponíveis no município, seja sob a forma de levantamentos epidemiológicos ou inquéritos, como aplicado nesta pesquisa. Ademais, o tempo médio de cobertura encontrado para as áreas cobertas pela equipe de saúde bucal foi de 3,22 anos.

Nos resultados obtidos neste estudo, o percentual de entrevistados que compõem o grupo "não-assistido", independentemente do modelo de assistência odontológica, ultrapassa $63 \%$, com variações entre 62,9\% (adolescentes) e $69,5 \%$ (adultos), o que pode ser considerado alto. Esses valores, entretanto, não surpreendem quando os comparamos com pesquisas de base populacional mais recentes e que utilizaram metodologia semelhante, como o Projeto SB Brasil 30 e a PNAD 2003 27. De acordo com os dados do IBGE, em 2003, 65,9\% da população representativa para o Estado do Rio Grande do Norte não haviam consultado cirurgião-dentista nos 12 meses anteriores à pesquisa, cuja média nacional era de $61,2 \%$. Os dados do Projeto SB Brasil 30 também mostram semelhanças, indicando que, no Município de Natal, no ano de 2002, 53,3\% dos entrevistados não tiveram acesso ao cirurgião-dentista no último ano. Entretanto, desagregando esse dado por tipo de serviço procurado, observa-se que o percentual sobe para $63,5 \%$ quando avaliamos somente a procura pelo serviço público, valor também muito próximo da média nacional de $62,1 \%$.

Esses dados, em certa medida, reforçam a qualidade da informação coletada neste estudo e demonstram, também, que o município analisado não é muito diferente dos padrões nacionais de acesso a serviços.

Contudo, a grande questão na qual se debruçou esta pesquisa diz respeito ao possível efeito que uma estratégia como a inclusão das equipes de saúde bucal no PSF poderia gerar sobre esses indicadores. O Município de Natal tinha, à época 
em que os dados foram coletados, 110 equipes de saúde da família, cobrindo $47,44 \%$ da população, e 94 equipes de saúde bucal com uma cobertura de $40,54 \%$, representando aproximadamente 80 mil famílias cobertas pelas equipes de saúde bucal. Essas equipes de saúde bucal estavam distribuídas entre os quatro Distritos Sanitários, com uma concentração maior nos distritos norte e oeste, justificada pelo fato de serem os dois distritos com os piores indicadores sociais da cidade e com um tempo médio de implantação de três anos, conforme destacamos anteriormente.

Nessas áreas, a população tem disponível equipes com perfil diferenciado, em que o processo de trabalho é (ou, pelo menos deveria ser) mais adequado. Essas equipes têm melhor salário, trabalham maior número de horas, se qualificaram e respondem por uma estratégia que se propõe a reestruturar a atenção básica sob novas bases, com acolhimento, vínculo, responsabilização e centrada na família.

É esperado que, decorridos três anos, essas áreas tivessem melhores indicadores, particularmente os de acesso, como os aqui pesquisados. Fica claro, portanto, o efeito positivo da saúde bucal no PSF, nesse tipo de comparação.

Contudo, quando as áreas cobertas pelo modelo 1 são comparadas com as cobertas por unidades de saúde, cuja única diferença é o fato de não terem a presença de equipe de saúde bucal (modelo 2), o efeito se inverte. Em outras palavras, o acesso à assistência odontológica é mais facilitado em áreas cobertas pelo modelo 2, traduzindo-se em uma RP de 0,71 indicando "efeito protetor".

É possível inferir, valendo-se desses dados, que o impacto da saúde bucal no PSF só é significativo quando tomamos como base áreas sem nenhuma cobertura ou quando são cobertas por unidades tradicionais, sem PSF e nem PACS. Em certa medida, esse tipo de análise "isola" o efeito da presença da equipe de saúde bucal na unidade de saúde e fica demonstrado que esta presença não afeta o acesso à assistência odontológica.

Uma primeira hipótese para a ocorrência desse quadro é que a inclusão das equipes de saúde bucal nas unidades de saúde da família não se traduziram em uma mudança no processo de trabalho. Ao que parece, a prática das equipes de saúde bucal continua se dando no modo tradicional, com baixo impacto, baixa cobertura e um processo de trabalho ainda pautado na abordagem curativo-reparadora, embora este estudo não reúna dados suficientes para estabelecer conclusões neste sentido, pois este não foi seu objetivo 20,21,22.

Entretanto, um outro dado que chama atenção e que reforça esse raciocínio, é quando fra- cionamos essa análise a partir da modalidade de cobertura e faixa etária. Como claramente demonstrado na Figura 3, o modelo 1 supera todos os outros somente quando consideramos o grupo de menores de 12 anos. Isso faz com que as diferenças em relação ao modelo 2 sejam piores ainda nas faixas etárias de adultos e de idosos. Isso denota uma manutenção de estratégias tradicionais de priorização das ações de saúde bucal voltadas para crianças, caracterizando o modelo de SB-PSF em Natal como "escolar-sespiano", utilizando a expressão proposta por Zanetti et al. 31. Trata-se de uma abordagem sem base epidemiológica, uma vez que dados do Projeto SB Brasil para o Nordeste indicam que, considerando o grupo até 19 anos, as necessidades de tratamento para cárie dentária no grupo até 12 anos representam $47 \%$ do total 30 . Desse modo, $53 \%$ das necessidades estão concentradas no grupo de 13 a 19 anos, exatamente o de menor possibilidade de acesso. Trata-se, pois, de uma inversão de prioridades e é importante ressaltar que está em discussão apenas as necessidades básicas relativas à cárie dentária.

Esse processo de incorporação gradativo da equipe de saúde bucal no PSF provavelmente é o responsável, em parte, pela produção ambulatorial em Odontologia, que segundo registro do Sistema de Informações Ambulatoriais (SIA/ SUS, 2006. Departamento de Informática do SUS; http:/ / www.datasus.gov.br), apresenta uma proporção de procedimentos preventivos individuais e coletivos com relação ao total de ações ambulatoriais da ordem de $30 \%$ a $48 \%$ entre janeiro e setembro.

De fato, em estudo realizado no mesmo município por Almeida 22, foi observado que os cirurgiões-dentistas do PSF de Natal realizavam atividades preventivas em âmbito individual e coletivo, predominando no primeiro orientação de higiene bucal e aplicação tópica de flúor, enquanto que, no segundo, predominava a aplicação tópica de flúor em escolares. Apesar do PSF trabalhar com a família, existia uma ênfase na fluorterapia, reproduzindo ações do movimento preventivista ${ }^{32}$. Outra conclusão que chamou a atenção foi o fato de ter havido maior regularidade de ações nos grupos de escolares e gestantes. Ainda segundo a autora, o repertório de atividades preventivas realizadas pelos cirurgiões-dentistas pertencentes ao PSF reproduz, de um modo geral, o modelo tradicional. Nas suas palavras, "verifica-se atuação em diferentes grupos, mas com maior atenção a escolares, assim como direcionamento das atividades à cárie dentária, existindo pouca ênfase a outros problemas bucais". 
Percentual de indivíduos que referiram acesso à assistência odontológica de acordo com o grupo etário. Natal, Rio Grande do Norte, Brasil, 2006

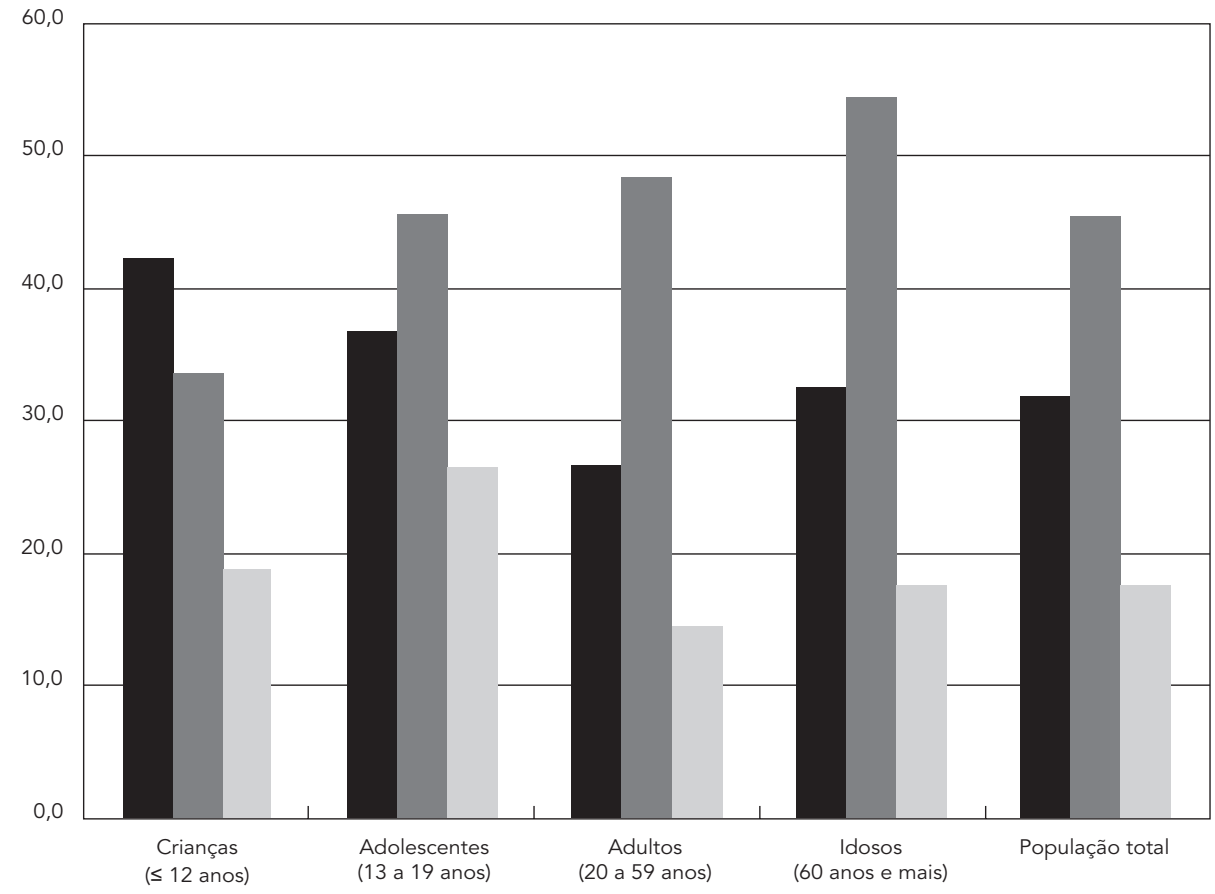

\section{Considerações finais}

Sem dúvida, o PSF se constitui em uma estratégia ímpar na reorganização da atenção básica no Brasil, e diversos estudos 13,33,34,35,36 têm demonstrado isto desde a sua implantação oficial a partir de 1994 .

O presente trabalho, feito em apenas um município de grande porte, capital de um estado do Nordeste, não demonstra a ausência de efeito da SB-PSF per se. Demonstra que a maneira como as estratégias são implantadas nos municípios são cruciais para o seu sucesso. O modo como se estabeleceu o processo de consolidação da Reforma Sanitária Brasileira - cujo eixo assistencial é representado pelo SUS - apostou radicalmente na municipalização como a melhor maneira para se atingir seu ideário de universalização, integralidade, eqüidade e controle social. Se por um lado temos hoje um dos mais significativos processos de descentralização em todo o mundo, por outro ainda persistem modelos assistenciais das mais distintas formas, espalhados pelos mais de 5.500 municípios do Brasil.

Assim, um grande processo de amadurecimento ainda é necessário para que políticas se traduzam em programas e modelos. E, mais ainda, que esses modelos impactem (duplamente) sobre o processo de trabalho e sobre as condições de saúde da população. 


\section{Resumo}

Este estudo objetivou verificar se a incorporação da equipe de saúde bucal no Programa Saúde da Família (PSF) gerou uma maior utilização dos serviços odontológicos no Município de Natal, Rio Grande do Norte, Brasil. Tomou-se como referência 22 setores censitários, 11 inseridos em áreas cobertas pela saúde bucal no PSF há pelo menos um ano, emparelhados com base em critérios sócio-econômicos, com 11 setores de áreas não-cobertas. A amostra final constou de 4.604 indivíduos. Foram comparadas áreas cobertas pela saúde bucal no PSF (modelo 1) com áreas cobertas por unidades de PSF porém sem saúde bucal (modelo 2) $e$ com áreas cobertas por unidades básicas de saúde tradicionais ou sem nenhuma cobertura (modelo 3). Os resultados mostraram que, nas áreas cobertas pelo modelo 1, 32\% referiram assistência odontológica, sendo este resultado igual a 45,4\% para o modelo 2 e 17,5\% para o modelo $3(p<0,001)$. Analisando-se as faixas etárias, o modelo 1 supera o modelo 2 apenas na faixa de até 12 anos. Conclui-se, portanto, que a saúde bucal no PSF apresenta impacto positivo sobre o indicador avaliado somente quando comparada com áreas sem cobertura (modelo 3).

Avaliação de Serviços de Saúde; Programa Saúde da Família; Saúde Bucal

\section{Colaboradores}

C. R. S. Pereira participou das etapas de análise dos dados, construção e redação final do texto. A. A. R. Patrício foi responsável pela concepção do trabalho, coleta dos dados, análise e redação final do texto. F. A. C. Araújo e E. E. S. Lucena participaram da elaboração dos desenhos amostrais, da coleta e análise dos dados e redação final do texto. K. C. Lima e A. G. Roncalli foram responsáveis pela concepção do trabalho, elaboraram o desenho amostral, participaram da análise dos dados e redação final do texto.

\section{Agradecimentos}

Os autores agradecem às instituições financiadoras: Conselho Nacional de Desenvolvimento Científico e Tecnológico (CNPq pelo fomento) e Coordenação de Aperfeiçoamento de Pessoal de Nível Superior (CAPES pela bolsa) e à Secretaria Municipal de Saúde de Natal pelo apoio à realização da pesquisa.

\section{Referências}

1. Silva Jr. AG, Mascarenhas MTM. Avaliação da atenção básica em saúde sob a ótica da integralidade: aspectos conceituais e metodológicos. In: Alves DS, Guljor AG, organizadores. Cuidado: as fronteiras da integralidade. São Paulo: Editora Hucitec/ Rio de Janeiro: ABRASCO; 2004. p. 241-55.

2. Merhy E, Franco TB. Programa Saúde da Família: somos contra ou a favor? Saúde Debate 2002; 26:118-22.

3. Ronzani TM, Stralen CJ. Dificuldades de implantação do Programa Saúde da Família como estratégia de reforma do sistema de saúde brasileiro. Rev APS 2003; 6:7-22.

4. Ministério da Saúde. Portaria no ${ }^{\circ}$ 1.444/GM. Cria o incentivo de Saúde Bucal para o financiamento de ações e da inserção de profissionais de Saúde Bucal no Programa de Saúde da Família. Diário Oficial da União 2000; 29 dez.

5. Ministério da Saúde. Portaria nº. 267. Aprova as Normas e Diretrizes de Inclusão da Saúde Bucal na Estratégia do Programa de Saúde da Família (PSF). Diário Oficial da União 2001; 7 mar.
6. Ministério da Saúde. Portaria no. 74. Reajusta os valores dos incentivos financeiros às ações de saúde bucal no âmbito do Programa Saúde da Família, inclui procedimento de moldagem para prótese e dá outras providências. Diário Oficial da União 2004; 22 jan.

7. Ministério da Saúde. Consolidado de equipes de saúde bucal e equipes de saúde da família - janeiro/2008. http://www.saude.gov.br/bucal (acessado em 20/Fev/2008).

8. Chaves SCL, Vieira-da-Silva LM. Atenção à saúde bucal e a descentralização da saúde no Brasil: estudo de dois casos exemplares no Estado da Bahia. Cad Saúde Pública 2007; 23:1119-31.

9. Roncalli AG. A organização da demanda em serviços públicos de saúde bucal: universalidade, eqüidade e integralidade em saúde bucal coletiva [Tese de Doutorado]. Araçatuba: Faculdade de Odontologia, Universidade Estadual Paulista Júlio de Mesquita Filho; 2000. 
10. Peres MAA. A saúde bucal em um sistema local de saúde: estudo de caso no Município de Ipatinga, MG, Brasil 1989-1994 [Dissertação de Mestrado]. São Paulo: Faculdade de Saúde Pública, Universidade de São Paulo; 1995.

11. Werneck MAF. A saúde bucal no SUS: uma perspectiva de mudança [Tese de Doutorado]. Niterói: Universidade Federal Fluminense; 1994.

12. Serra CG. A saúde bucal como política de saúde: análise de três experiências recentes: Niterói, Campinas e Curitiba [Dissertação de Mestrado]. Rio de Janeiro: Instituto de Medicina Social, Universidade do Estado do Rio de Janeiro; 1998.

13. Bodstein, R, Feliciano K, Hortale VA, Leal, MC. Estudos de linha de base do Projeto de Expansão e Consolidação do Saúde da Família (ELB/Proesf): considerações sobre seu acompanhamento. Ciênc Saúde Coletiva 2006; 11:725-31.

14. Baldani MH, Fadel CB, Possamai T, Queiroz MGS. A inclusão da odontologia no Programa Saúde da Família no Estado do Paraná, Brasil. Cad Saúde Pública 2005; 21:1026-35.

15. Andrade KLC, Ferreira EF. Avaliação da inserção da odontologia no Programa Saúde da Família de Pompeu (MG): a satisfação do usuário. Ciênc Saúde Coletiva 2006; 11:123-30.

16. Oliveira JLC, Saliba NA. Atenção odontológica no Programa Saúde da Família de Campos dos Goytacazes. Ciênc Saúde Coletiva 2005; 10:297-302.

17. Donabedian A. The role of outcomes in quality assessment and assurance. QRB Qual Rev Bull 1992; 18:356-60.

18. Draibe SM. Avaliação de implementação: esboço de uma metodologia de trabalho em políticas públicas. In: Barreira MCR, Carvalho MCB, organizadores. Tendências e perspectivas na avaliação de políticas e programas sociais. São Paulo: Instituto de Estudos Especiais, Pontifícia Universidade Católica de São Paulo; 2004. p. 15-42.

19. Szwarcwald CL, Viacava F, Vasconcellos MTL, Leal MC, Azevedo LO, Queiroz RSB, et al. Pesquisa Mundial de Saúde 2003. O Brasil em números. Radis $2004 ; 23: 14-34$.

20. Souza TMS, Roncalli AG. Saúde bucal no Programa Saúde da Família: uma avaliação do modelo assistencial. Cad Saúde Pública 2007; 23:2727-39.

21. Rocha RACP. Avaliação do acesso efetivo aos serviços odontológicos em áreas cobertas pela Estratégia Saúde da Família em Campina Grande - PB [Tese de Doutorado]. Recife: Universidade de Pernambuco; 2007.

22. Almeida GCM. Atividades preventivas realizadas pelos cirurgiões-dentistas do PSF de Natal/RN [Dissertação de Mestrado]. Natal: Universidade Federal do Rio Grande do Norte; 2007.
23. Travassos C, Martins M. Uma revisão sobre os conceitos de acesso e utilização de serviços de saúde. Cad Saúde Pública 2004; 20 Suppl 2:S190-8.

24. Almeida Filho N, Rouquayrol Z. Desenhos de pesquisa epidemiológica. In: Rouquayrol Z, Almeida Filho N, organizadores. Epidemiologia \& saúde. Rio de Janeiro: Editora Medsi; 1999. p. 149-70.

25. Instituto Brasileiro de Geografia e Estatística. Base de dados por setor censitário [CD-ROM]. Rio de Janeiro: Instituto Brasileiro de Geografia e Estatística; 2005.

26. Roncalli AG, Lima KC. Impacto do Programa Saúde da Família sobre indicadores de saúde da criança em municípios de grande porte da região Nordeste do Brasil. Ciênc Saúde Coletiva 2006; 10:297-302.

27. Instituto Brasileiro de Geografia e Estatística. Pesquisa Nacional por Amostra de Domicílios: acesso e utilização de serviços de saúde, 2003. Rio de Janeiro: Instituto Brasileiro de Geografia e Estatística; 2005.

28. Altman DG. Practical statistics for medical research. New York: Chapman \& Hall; 1990.

29. Luiz RR. Análise estatística com múltiplas variáveis. In: Luiz RR, Costa AJL, Nadanovsky P, organizadores. Epidemiologia e bioestatística na pesquisa odontológica. São Paulo: Editora Atheneu; 2005. p. 341-72.

30. Coordenação Nacional de Saúde Bucal, Departamento de Atenção Básica, Secretaria de Atenção à Saúde, Ministério da Saúde. Projeto SB Brasil 2003: condição de saúde bucal da população brasileira, 2002-2003. Brasília: Ministério da Saúde; 2004.

31. Zanetti CHG, Lima MAU, Ramos L, Costa MABT Em busca de um paradigma de programação local em saúde bucal mais resolutivo no SUS. Divulg Saúde Debate 1996; 13:18-35.

32. Cordón J, Garrafa V. Prevenção versus preventivismo. Divulg Saúde Debate 1991; 8:10-6.

33. Ministério da Saúde. Avaliação da implementação do Programa Saúde da Família em 10 centros urbanos. Brasília: Ministério da Saúde; 2002.

34. Henrique F, Calvo MCM. Avaliação do Programa Saúde da Família nos municípios do Estado de Santa Catarina, Brasil. Cad Saúde Pública 2008; 24:809-19.

35. Canesqui AM, Spinelli MAS. A implementação do Programa Saúde da Família em municípios do Estado de Mato Grosso, Brasil. Cad Saúde Pública 2008; 24:862-70.

36. Vieira-da-Silva LM, Hartz ZMA, Chaves SCL, Silva GAP, Paim JS. Análise da implantação da gestão descentralizada em saúde: estudo comparado de cinco casos na Bahia, Brasil. Cad Saúde Pública $2007 ; 23: 355-70$

Recebido em 28/Ago/2008

Versão final reapresentada em 05/Set/2008 Aprovado em 08/Dez/2008 